OPEN ACCESS

Edited by:

Kristina Bertl,

Malmö University, Sweden

Reviewed by:

Olivier Huck

Université de Strasbourg, France

Daiane Cristina Peruzzo,

São Leopoldo Mandic School, Brazil

*Correspondence:

Ulrich Schlagenhauf

schlagenha_u@ukw.de

Specialty section:

This article was submitted to

Periodontics,

a section of the journal

Frontiers in Dental Medicine

Received: 12 May 2021

Accepted: 06 August 2021

Published: 03 September 2021

Citation:

Schlagenhauf $U$ and Jockel-Schneider Y (2021) Probiotics in the Management of Gingivitis and

Periodontitis. A Review.

Front. Dent. Med. 2:708666.

doi: 10.3389/fdmed.2021.708666

\section{Probiotics in the Management of Gingivitis and Periodontitis. A Review}

\author{
Ulrich Schlagenhauf $^{1,2 *}$ and Yvonne Jockel-Schneider ${ }^{1}$ \\ ${ }^{1}$ Department of Conservative Dentistry and Periodontology, Center for Oral Health, University Hospital Würzburg, Würzburg, \\ Germany, ${ }^{2}$ Clinic of Conservative and Preventive Dentistry, Division of Periodontology and Peri-implant Diseases, Center of \\ Dental Medicine, University of Zurich, Zurich, Switzerland
}

In the management of intestinal health problems, the targeted use of probiotic microorganisms is a common therapeutic measure with a long-standing tradition. In clinical dentistry however, probiotics-based therapy is still a rather new and developing field, whose usefulness for the control of gingivitis and periodontitis has been questioned by recent meta-analyses and systematic reviews. The purpose of the subsequent descriptive review is to provide an introduction to the concept of probiotic microorganisms and their multifaceted health-promoting interactions with the human host and microbial competitors, followed by a detailed comparison of the results of available controlled clinical trials assessing the use of probiotics in the control of gingival and periodontal inflammations. It aims at contributing to a deeper understanding of the unique capabilities of probiotics to resolve chronic plaque-induced inflammation even in the absence of mechanical plaque control and will discuss how possible misconceptions about the rationale for using probiotics may have led to the present controversies about their usefulness as a therapeutic option.

Keywords: probiotics, gingivitis, periodontitis, review, periodontal therapy

\section{THE HUMAN MICROBIOME}

The skin and all mucosal surfaces of the human body are colonized by a multitude of different microbial species. According to current estimates (1) their cumulative number $\left(3.0 \times 10^{13}\right)$ roughly matches the number of human body cells. Due to the enormous species richness of colonizing microorganisms their combined genome has been recently calculated to contain a total of more than 45 million different microbial genes (2). It vastly outnumbers the human genome, containing, depending on the definition, only about 20.000-100.000 different genes (3), which has contributed to the understanding, that host-microbiota interactions are an integral and essential part of human physiology (4). While the concept of antisepsis pioneered by Louis Pasteur and Robert Koch certainly laid the foundation for the control of infectious diseases and saved the lives of millions of people, it narrowed the focus of scientific efforts for many decades mainly to the exploration of bacterial pathogens, which in a healthy host are either absent or forming only a minute portion of the resident microbiota. This is particularly true in clinical dentistry, where the significance of the oral microbiota still is mostly reduced to being a health threat, exemplified by the fact, that therapeutic concepts based on the indiscriminate elimination of oral bacterial biofilms continue to dominate even the most recent evidence-based guidelines for the management of caries and periodontal disease (5-7). 


\section{Microorganisms With a Beneficial Impact on the Health of Their Host (Probiotics)}

Nevertheless, in some Asian countries like Japan, the beneficial impact of the consumption of certain bacterially fermented foods on human health has been known already as experiential knowledge for centuries. Nobel laureate Eli Metchnikoff was the first modern-day scientist, who investigated the nature of beneficial bacteria systematically (8). Noting that Bulgarian peasants lived longer than those in other countries, despite comparably miserable living conditions, he traced down the possible reason for the observed longevity to the consumption of yogurt, which was very popular in Bulgaria and attributed its health-promoting effect to its rich content of the bacterial species Lactobacillus delbrueckii. subsp. bulgaricus. Ever since a whole variety of beneficial bacteria and yeasts, mostly associated with fermented food components of the human diet has been identified and is nowadays summarized by the term "probiotics." According to a definition set by the World Health Organization in 2001 (9), probiotics are microorganisms, which survive the passage through the acidity of the stomach alive, and when consumed in adequate amounts, confer a health benefit on the host. This definition has been met so far by a series of microorganisms among them e.g., various strains of Lactobacillus spp., Streptococcus spp., Bifidobacterium spp., Bacillus spp. as well as the Escherichia coli strain Nissle 1917 or the yeast Saccharomyces boulardii (10). Their health-promoting probiotic properties may originate from a local interference with competing members of the human microbiota as well as by a direct contact to cellular structures of the mucosal immune system of the host, which may not only elicit a local but also a systemic response modulating inflammatory reactions even in distant parts of the body. As schematically depicted in Figure 1, probiotics are able to inhibit other microorganisms competing for the same space and/or substrates by interfering with their metabolism and/or living conditions. This may occur by a variety of measures, like a change in local $\mathrm{pH}$ or the concentration of reactive oxygen or nitrogen species (ROS; RN) via the synthesis of metabolites like organic acids, peroxide or nitric oxide $(11,12)$ as well as by the synthesis and release of so called bacteriocines, specific peptides, able to selectively kill competing bacteria or to change the spectrum of their metabolism (13-15). Some probiotics are also capable to successfully impair the biofilm formation of their microbial competitors by various mechanisms including the quenching of their quorum sensing communication $(16,17)$. Many probiotic bacteria and particularly probiotic lactobacilli display the ability to attach to the mucus lining of the gut and oral epithelia (18), which brings them in close proximity to the body cells. Their health-promoting impact therefore may also arise from a selective interference with immunocompetent host cells like dendritic cells, T-cells or generic epithelial cells at the interface between the lining mucosa and the opposing microbiota (see Figure 2). This may result in an improved epithelial barrier function due to a stimulated increase in the thickness of the protective mucus layer as well as due to reestablishing the tightness of damaged interepithelial tight junctions $(19,20)$.
Moreover, the consumption of probiotic Lactobacillus reuteri strains showed to enhance tissue repair of defined skin wounds in mice as well as in human volunteers despite not being physically in direct contact with the wound, by increasing the plasma level of the neuropeptide oxytocin $(21,22)$, accompanied by a downregulation of blood corticosterone levels and a reduction in the number of circulating blood neutrophils. In an in vitro experiment the exposure of mouse bone marrow dendritic cells (DC) to L. reuteri supernatants or sterile L.reuteri-lysates promoted DC maturation and upregulated the secretion of antiinflammatory interleukin-10 (23). In a rat model of necrotizing enterocolitis, which is also a life-threatening condition in the intestine of premature human infants, the addition of L.reuteri DSM 17938 to the diet of the newborn rats resulted in a downregulation of enteric inflammation by an increased migration of Foxp3(+) regulatory T-cells to the inflamed parts of the gut (24). Immunomodulatory properties however are not limited to specific $L$. reuteri strains but have also been identified in many other commonly used probiotics (25). While various probiotic bacteria readily adhere to the intestinal and oral epithelia after ingestion, it must be noted, that most of them are unable to colonize their new host permanently (26). Clinical trials have shown that the number of detectable probiotic bacteria rapidly decreases within days to few weeks after the cessation of their consumption, making their continuously repeated intake for the maintenance of the beneficial effects associated with them a necessity (27). Although most therapeutically used probiotic bacteria have been initially retrieved from the resident microbiota of healthy human individuals, they may not readily fit into the microbial spectrum of other human hosts due to inhibition by microbial competitors from the resident host microbiota, an insufficient genetic adaption to the new host or, probably most important, due to a lack of suitable growth substrates provided by the daily diet of their actual host, as indicated by studies evaluating the impact of different food elements on the growth and the diversity of the human microbiota $(28,29)$.

\section{Safety Aspects of Probiotics Consumption}

Given the wide array of known metabolic interferences with the human microbiota and the impact on the physiology of the host as described before, potential adverse effects associated with an indiscriminate, medically unsupervised consumption of probiotics need to be considered. They include the induction of bacterial translocation, bacteremia, sepsis, bacterial dysbiosis and the conferral of bacterial resistance genes, further complicated by the development of systemic health problems induced by these conditions (30). Cumulative evidence from the literature nevertheless, proves an overall excellent safety record for the long-term consumption of probiotics by healthy adults and children (31-33). There are however various case reports of bacteremia, fungemia or bacterial dysbiosis or other adverse events. It has to be noted, that the vast majority of them occurred in individuals, who were concomitantly affected by severe chronic systemic disease or compromised immunity and/or frailty $(32,34-37)$, conditions also frequently associated 


\section{Specific Interference}

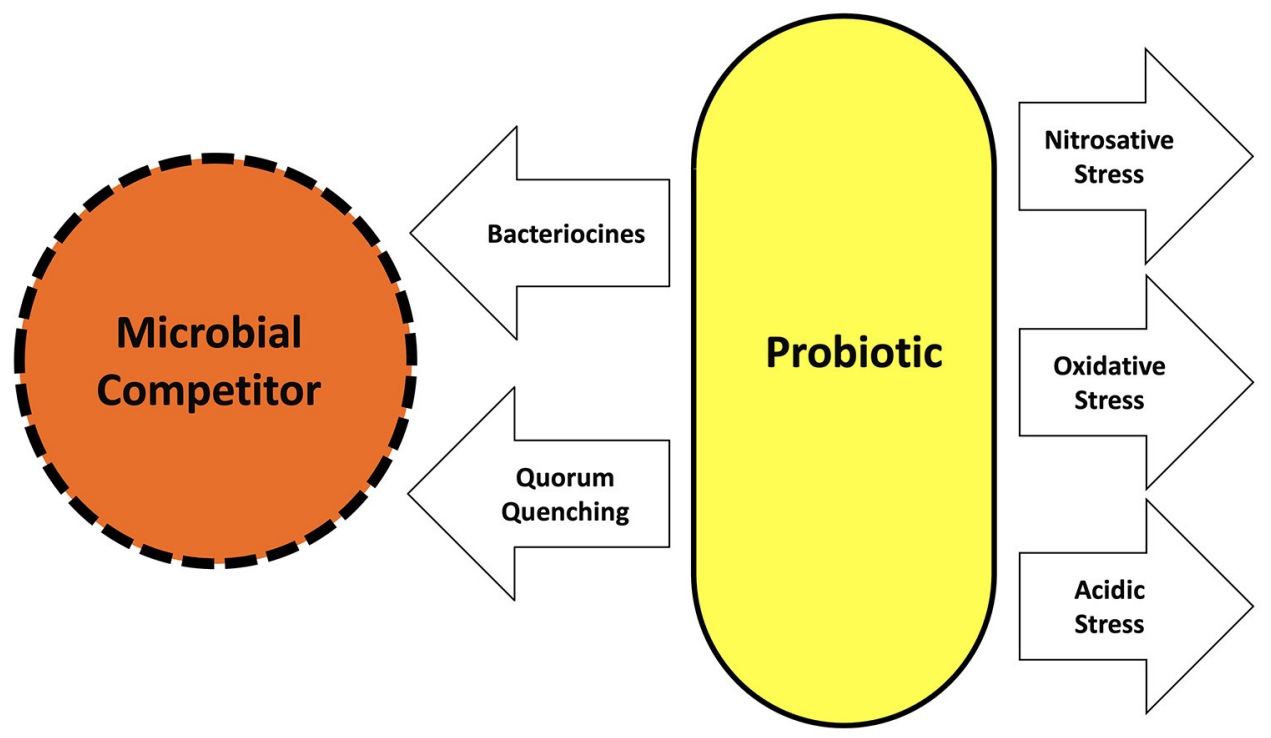

Unspecific Interference

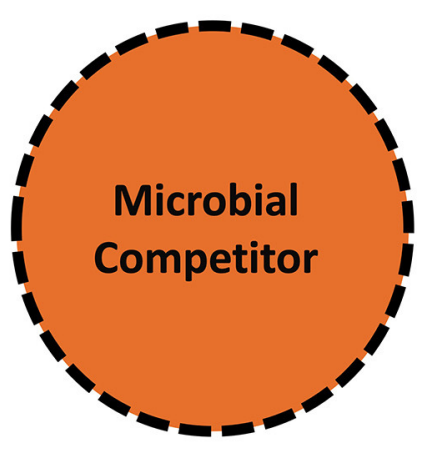

FIGURE 1 | Mechanisms used by probiotics to interfere with the growth and metabolism of competing microorganisms.

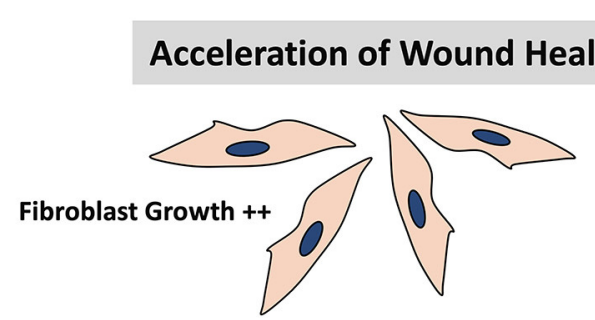

Stimulation of Systemic Oxytocin Release

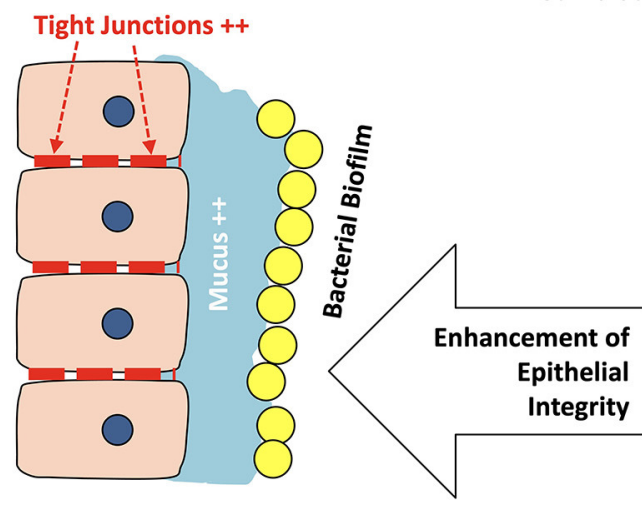

Inhibition of Microbial Invasion
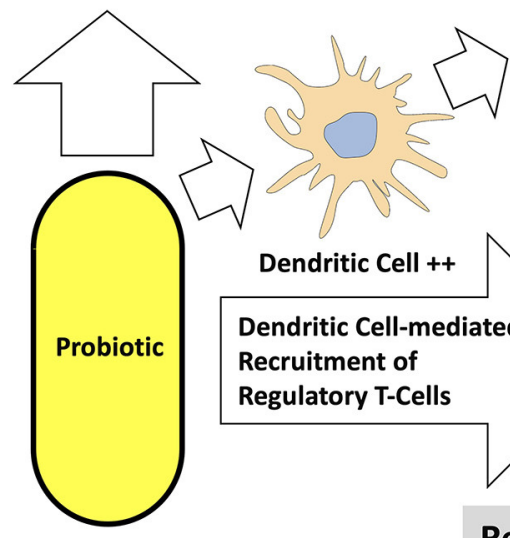

Interleukin 10-mediated Inhibition

of Proinflammatory Th17 Cells
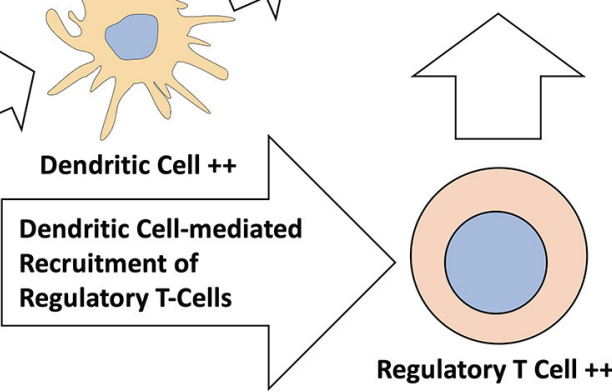

Reduction of Inflammation

$\leftrightarrow \odot \odot$

FIGURE 2 | Mechanisms used by probiotics to interfere with their host locally and on a systemic level.

with bacterial dysbioses of the human microbiome (38) and the development of severe periodontitis (39-42). The validity of a further, much-noticed case report blaming the development of Dlactic acidosis-induced "brainfogginess" to the consumption of a mix of lactobacilli and bifidobacteria, Streptococcus thermophilus and some other unspecified bacterial species (43) has been challenged by other experts, as all commonly used probiotic lactobacilli and bifidobacteria are known to exclusively synthesize 
L-lactic acid (44). In a clinical study, assessing urinary Dlactic acid concentrations in healthy newborns, who consumed a L.reuteri DSM 17938-containing infant formula during the first 28 days of their life, the addition of the probiotic to their diet did not initiate or facilitate the colonizing of D-lactic acid synthesizing bacteria in the developing intestinal microbiota of the newborns, compared to controls, consuming an identical infant formula being void of probiotics (45). Another widespread misunderstanding is the assumption, that probiotics may only be consumed for a limited time. This may have arisen because the duration of probiotic consumption reported in clinical trials is usually only weeks or a few months. However, all commercially available probiotic products must have approval from the relevant regulatory authorities as food supplements, which includes the safety of indefinite, long-term consumption (see next section legal and commercial aspects). In summary the consumption of probiotics may be regarded safe for systemically healthy persons, but it may be advisable to use only probiotic products whose safety profile for the intended application purpose has been verified by in vitro assessments and clinical trials (30).

\section{Legal and Commercial Aspects of Probiotics}

In the European Union and the United States probiotics are not legally considered medicines but food supplements. Their market admission therefore needs to comply with the safety regulations of the European Food Safety Authority (EFSA) or the US Food and Drug Administration (FDA) for a food or food supplement, but it is not dependent on the verified proof of any health benefit. Currently there is a multitude of commercial probiotic food supplements freely available, which all meet EFSA and/or FDA safety standards, but which may not have any evidence for a health-promoting impact supported by the results of randomized controlled clinical trials. As a matter of fact, in 2011 EFSA did no longer allow the promotion of any health claim for any commercial probiotic product sold in Europe, due to a general lack of scientific evidence meeting the efficacy standards set by EFSA (EC regulations \#1924/2006, \# 1169/2011). It must be noted in this context, that the extent and the specificity of the health-promoting efficacy of probiotics show huge variations even within different strains of the same species. Therefore, the consumption of two seemingly comparable probiotic food supplements containing different strains of the same bacterial species may show profound differences regarding their impact on the microbiota as well as on the health status of their host $(30,46)$. Also, the indiscriminate use of probiotic multi-species food supplements with possibly opposing properties may not be advisable due to the arising complexity of interactions (30).

\section{PROBIOTICS IN THE MANAGEMENT OF ORAL HEALTH PROBLEMS}

Although the vast majority of available scientific evidence regarding the therapeutic usefulness of probiotics has originated so far from the field of intestinal health research, there is now a steadily growing number of randomized controlled clinical trials (RCT) dedicated to the evaluation of probiotics in the management of oral health problems and in particular gingivitis and periodontitis. The subsequent descriptive analysis is based on the results of a total of 36 available RCTs, evaluating the use of probiotics in the control of gingivitis or periodontitis. They were published between 2009 and 2021 and were all retrieved from the PubMed database. Their main characteristics are schematically listed in Tables 1, 2.

\section{Probiotics Evaluated in Oral Trials}

The most frequently evaluated probiotic, used in 17 of the reviewed 36 RCTs, was a combination of two probiotic Lactobacillus reuteri strains (DSM17938; ATCC PTA 5289) (49-54, 67-76), followed by various probiotic Lactobacillus rhamnosus strains (60-62, 79-81), probiotic strains of Lactobacillus brevis (56, 57, 77), Bifidobacterium animalis subsp. lactis (48, 64, 65), Lactobacillus casei Shirota $(58,59)$, Weissella cibaria (63), Lactobacillus plantarum (78) and combinations of probiotic Bacillus subtilis, B. megaterium und B. pumilus (47), Streptococcus oralis, S. uberis and S. rattus (82) as well as Streptococcus thermophilus, Enterococcus faecium, Bifidobacterium longum, and B. animalis subsp. lactis strains in conjunction with prebiotic fructooligosaccharides (55).

\section{Application, Dosage and Duration of Consumption}

In most trials the probiotic bacteria were consumed as an ingredient of a lozenge, but also the administration via a probiotic yogurt $(58,59)$ or as an ingredient of toothpaste $(47)$ has been evaluated. The reported probiotics dosage administered in a single application mostly varied between $10^{8}$ and $10^{9}$ colony forming units (CFU), with a bandwidth ranging from as low as $3 \times 10^{3} \mathrm{CFU}(57)$ up to $6.5 \times 10^{9} \mathrm{CFU}$ (58). The number of daily intakes varied between $1 \times$ daily to $4 \times$ daily, with $2 \times$ daily being the most frequently specified consumption scheme. Total duration of consumption varied from 21 to 84 days.

\section{Probiotics in the Management of Chronic Gingivitis and Experimental Gingivitis Evaluated Study Cohorts}

The impact of probiotics administration in the management of chronic and experimental gingivitis has been assessed in a broad range of different cohorts, like adolescents $(48,61)$, adults $(56,57,59,62)$, dental students $(49,58,63)$, pregnant women (52), smokers (55), navy sailors on duty (54), diabetics (53), or frail elderly (51).

\section{Evaluated Study Outcomes}

Evaluated study outcomes were mostly clinical indices of gingival inflammation like gingival index (GI) and bleeding on probing $(\mathrm{BoP})$, often supplemented by the documentation of plaque accumulation and an analysis of the composition of the gingivitis-associated microbiota. Study participants were either affected by established chronic gingivitis of varying severity or were subjected to the development of experimental gingivitis after cessation of personal oral hygiene $(48,50,56,58,59)$. 
TABLE 1 | Randomized controlled trials evaluating the use of probiotics in the management of gingivitis.

\begin{tabular}{|c|c|c|c|c|c|c|c|c|c|c|c|c|c|c|c|}
\hline References & $\begin{array}{l}\text { Type of } \\
\text { investigation }\end{array}$ & $\begin{array}{l}\text { Assessed } \\
\text { condition }\end{array}$ & $\begin{array}{l}\text { No. } \\
\text { subjects }\end{array}$ & $\begin{array}{l}\text { No. } \\
\text { groups }\end{array}$ & PMPR & $\begin{array}{l}\text { Hygiene } \\
\text { instructions }\end{array}$ & Assessed probiotics & $\begin{array}{l}\text { Application } \\
\text { mode }\end{array}$ & $\begin{array}{l}\text { Dosage } \\
\text { (CFU) }\end{array}$ & $\begin{array}{l}\text { Frequency/ } \\
\text { day }\end{array}$ & $\begin{array}{l}\text { Duration } \\
\text { of intake }\end{array}$ & $\begin{array}{l}\text { Observation } \\
\text { time }\end{array}$ & $\begin{array}{l}\text { Assessed } \\
\text { Outcomes }\end{array}$ & $\begin{array}{l}\text { Primary } \\
\text { outcome }\end{array}$ & $\begin{array}{l}\text { Outcomes } \\
\text { significantly } \\
\text { improved by } \\
\text { consumption } \\
\text { of probiotic }\end{array}$ \\
\hline $\begin{array}{l}\text { Alkaya et al. } \\
\text { (47) }\end{array}$ & $\mathrm{RCT}$ & Gingivitis & 30 & 2 & Yes & Yes & $\begin{array}{l}\text { Bacillus subtills, Bacillus } \\
\text { megaterium, Bacillus pumilus }\end{array}$ & Toothpaste & $5 \times 10^{7}$ & $2 \times$ daily & 56 days & 56 days & $\begin{array}{l}\text { Pl, Gl, PPD, } \\
\text { BoP }\end{array}$ & $\mathrm{Pl}, \mathrm{Gl}$ & None \\
\hline Kuru et al. (48) & RCT & $\begin{array}{l}\text { Experimental } \\
\text { gingivitis }\end{array}$ & 51 & 2 & Yes & Yes & $\begin{array}{l}\text { Bifidobacterium.animalis.subsp. } \\
\text { lactis DN-173010 }\end{array}$ & Yogurt & $1 \times 10^{8}$ & $1 \times$ daily & 28 days & 33 days & $\begin{array}{l}\text { GCF volume, } \\
\text { PI, GI PPD, } \\
\text { BoP }\end{array}$ & Not specified & $\begin{array}{l}\mathrm{Pl} \text {, Gl, BoP, } \\
\text { GCF volume }\end{array}$ \\
\hline $\begin{array}{l}\text { Iniesta et al. } \\
\text { (49) }\end{array}$ & $\begin{array}{l}\text { RCT } \\
\text { Cross-over\# }\end{array}$ & Gingivitis & 40 & 2 & Yes & No & $\begin{array}{l}\text { Lactobacillus reuteri DSM } \\
\text { 17938/ATCC PTA } 5289\end{array}$ & Lozenge & $2 \times 10^{8}$ & $1 \times$ daily & 28 days & 28 days & $\mathrm{Gl}, \mathrm{Pl}$ & Not specified & None \\
\hline $\begin{array}{l}\text { Hallström et al. } \\
\text { (50) }\end{array}$ & $\begin{array}{l}\text { RCT } \\
\text { Cross-over }\end{array}$ & $\begin{array}{l}\text { Experimental } \\
\text { gingivitis }\end{array}$ & 18 & 2 & Yes & Yes & $\begin{array}{l}\text { Lactobacillus reuteri DSM } \\
\text { 17938/ATCC PTA } 5289\end{array}$ & Lozenge & $2 \times 10^{8}$ & $2 \times$ daily & 21 days & 21 days & $\mathrm{Pl}, \mathrm{Gl}, \mathrm{BoP}$ & Not specified & None \\
\hline $\begin{array}{l}\text { Kraft-Bodi } \\
\text { et al. (51) }\end{array}$ & $\mathrm{RCT}$ & $\begin{array}{l}\text { Gingivitis } \\
\text { candidiasis }\end{array}$ & 215 & 2 & No & No & $\begin{array}{l}\text { Lactobacillus reuteri DSM } \\
\text { 17938/ATCC PTA } 5289\end{array}$ & Lozenge & $2 \times 10^{8}$ & $2 \times$ daily & 84 days & 84 days & $\begin{array}{l}\text { Candida count } \\
\mathrm{Pl}, \mathrm{BoP}\end{array}$ & Candida count & Candida count \\
\hline $\begin{array}{l}\text { Schlagenhauf } \\
\text { et al. (52) }\end{array}$ & RCT & $\begin{array}{l}\text { Gingivitis } \\
\text { pregnancy }\end{array}$ & 45 & 2 & No & No & $\begin{array}{l}\text { Lactobacillus reuteri DSM } \\
\text { 17938/ATCC PTA } 5289\end{array}$ & Lozenge & $2 \times 10^{8}$ & $2 \times$ daily & 49 days & 49 days & $\mathrm{Gl}, \mathrm{PI}$ & Gl & $\mathrm{Gl}, \mathrm{PI}$ \\
\hline $\begin{array}{l}\text { Sabatini et al. } \\
\text { (53) }\end{array}$ & $\begin{array}{l}\text { RCT No } \\
\text { Placebo }\end{array}$ & $\begin{array}{l}\text { Gingivitis } \\
\text { diabetes }\end{array}$ & 80 & 2 & No & Yes & $\begin{array}{l}\text { Lactobacillus reuteri DSM } \\
\text { 17938/ATCC PTA } 5289\end{array}$ & Lozenge & $2 \times 10^{8}$ & $2 x$ daily & 30 days & 30 days & $\mathrm{Pl}, \mathrm{BoP}$ & Not specified & $\mathrm{Pl}, \mathrm{BoP}$ \\
\hline $\begin{array}{l}\text { Schlagenhauf } \\
\text { et al. (54) }\end{array}$ & $\mathrm{RCT}$ & Gingivitis & 72 & 2 & No & No & $\begin{array}{l}\text { Lactobacillus reuteri DSM } \\
\text { 17938/ATCC PTA } 5289\end{array}$ & Lozenge & $2 \times 10^{8}$ & $2 \times$ daily & 42 days & 42 days & $\begin{array}{l}\text { BoP, PI, Gl, } \\
\text { PPD, CAL }\end{array}$ & BoP & $\begin{array}{l}\text { BoP, Pl, Gl, } \\
\text { PPD, CAL }\end{array}$ \\
\hline Ercan et al. (55) & RCT & $\begin{array}{l}\text { Gingivitis } \\
\text { smoking }\end{array}$ & 80 & 4 & Yes & Yes & $\begin{array}{l}\text { Lactobacillus acidophilus, } \\
\text { Bifidobacterium } \\
\text { animalis.subsp. lactis, } \\
\text { Enterococcus faecium, } \\
\text { Streptococcus thermophilus, } \\
\text { Bifidobacterium Iongum }\end{array}$ & Lozenge & $1.4 \times 10^{8}$ & $1 \times$ daily & 30 days & 30 days & $\begin{array}{l}\text { GCF } \\
\text { interleukins PI, } \\
\text { Gl }\end{array}$ & $\begin{array}{l}\text { GCF } \\
\text { interleukins }\end{array}$ & None \\
\hline Lee et al. (56) & $\mathrm{RCT}$ & $\begin{array}{l}\text { Experimental } \\
\text { gingivitis }\end{array}$ & 30 & 2 & Yes & Yes & Lactobacillus brevis CD2 & Lozenge & $1 \times 10^{9}$ & $3 \times$ daily & 14 days & 14 days & $\begin{array}{l}\text { Gl, Pl, BoP, } \\
\text { PPD GCF } \\
\text { volume }\end{array}$ & Not specified & None \\
\hline $\begin{array}{l}\text { Montero et al. } \\
\text { (57) }\end{array}$ & $\mathrm{RCT}$ & Gingivitis & 52 & 2 & Yes & Yes & $\begin{array}{l}\text { Lactobacillus brevis, } \\
\text { Lactobacillus plantarum CECT } \\
7481 \text { Pediococcus acidilactici } \\
\text { CECT } 8633\end{array}$ & Lozenge & $3 \times 10^{3}$ & $2 \times$ daily & 42 days & 42 days & $\mathrm{Gl}, \mathrm{PI}$ & Gl & high Gl scores \\
\hline $\begin{array}{l}\text { Staab et al. } \\
\text { (58) }\end{array}$ & $\mathrm{RCT}$ & $\begin{array}{l}\text { Experimental } \\
\text { gingivitis }\end{array}$ & 50 & 2 & No & No & Lactobacillus casei Shirota & Milk drink & $6.5 \times 10^{9}$ & 1 daily & 56 days & 60 days & $\begin{array}{l}\text { API, PI, PBI } \\
\text { serum marker }\end{array}$ & Not specified & None \\
\hline $\begin{array}{l}\text { Slawik et al. } \\
\text { (59) }\end{array}$ & $\mathrm{RCT}$ & $\begin{array}{l}\text { Experimental } \\
\text { gingivitis }\end{array}$ & 28 & 2 & No & No & Lactobacillus casei Shirota & Milk drink & $6.5 \times 10^{9}$ & $1 \times$ daily & 28 days & 14 days $^{\star}$ & $\begin{array}{l}\mathrm{Pl}, \mathrm{Gl}, \mathrm{BoP} \\
\text { GCF volume }\end{array}$ & Not specified & $\begin{array}{l}\text { BoP, GCF } \\
\text { volume }\end{array}$ \\
\hline $\begin{array}{l}\text { Toiviainen et al. } \\
\text { (60) }\end{array}$ & RCT & Gingivitis & 60 & 2 & No & No & $\begin{array}{l}\text { Lactobacillus rhamnosus GG } \\
\text { Bifidobacterium.animalis.subsp. } \\
\text { lactis BB-12 }\end{array}$ & Lozenge & $4 \times 10^{9}$ & $4 \times$ daily & 28 days & 28 days & $\begin{array}{l}\text { Mutans } \\
\text { Streptococci } \\
\text { PI, Gl }\end{array}$ & $\begin{array}{l}\text { Mutans } \\
\text { Streptococci }\end{array}$ & $\mathrm{Pl}, \mathrm{Gl}$ \\
\hline $\begin{array}{l}\text { Alanzi et al. } \\
(61)\end{array}$ & $\mathrm{RCT}$ & Gingivitis & 108 & 2 & No & Yes & $\begin{array}{l}\text { Lactobacillus rhamnosus GG } \\
\text { Bifidobacterium.animalis.subsp. } \\
\text { lactis BB-12 }\end{array}$ & Lozenge & $2 \times 10^{8}$ & $2 \times$ daily & 28 days & 28 days & $\mathrm{Gl}, \mathrm{Pl}$ & Gl & Gl \\
\hline Keller et al. (62) & RCT & Gingivitis & 47 & 2 & No & Yes & $\begin{array}{l}\text { Lactobacillus rhamnosus } \\
\text { PB01 DSM14869 } \\
\text { Lactobacillus curvatus EB10 } \\
\text { DSM32307 }\end{array}$ & Lozenge & $1 \times 10^{8}$ & $2 \times$ daily & 28 days & 42 days & $\begin{array}{l}\text { BoP, PI GCF } \\
\text { cytokines }\end{array}$ & Not specified & None \\
\hline Kang et al. (63) & $\mathrm{RCT}$ & Gingivitis & 68 & 2 & Yes & No & Weissella cibaria CMU & Lozenge & $1 \times 10^{8}$ & $1 \times$ daily & 56 days & 56 days & $\begin{array}{l}\text { PPD, BoP PI, } \\
\text { Gl }\end{array}$ & Not specified & None \\
\hline
\end{tabular}

API, Approximal Plaque Index; BoP, Bleeding on Probing; CAL, Clinical Attachment Level; Gl, Gingival Index; GCF, Gingival Crevicular Fluid; PI, Plaque Index; PPD, Probing Pocket Depth

"Due to s a significant order-of-use effect only data of the first cross-over period included in the analysis.

*Unsupervised consumption of the probiotic started 14 days before baseline. 
TABLE 2 | Randomized controlled clinical trials evaluating the use of probiotics in the management of periodontitis.

\begin{tabular}{|c|c|c|c|c|c|c|c|c|c|c|c|c|c|c|c|}
\hline References & $\begin{array}{l}\text { Type of } \\
\text { investigation }\end{array}$ & $\begin{array}{l}\text { Assessed } \\
\text { condition }\end{array}$ & $\begin{array}{l}\text { No. } \\
\text { subjects }\end{array}$ & $\begin{array}{l}\text { No. } \\
\text { groups }\end{array}$ & $\begin{array}{l}\text { Professional } \\
\text { scaling }\end{array}$ & $\begin{array}{l}\text { Hygiene } \\
\text { instructions }\end{array}$ & Assessed probiotics & $\begin{array}{l}\text { Application } \\
\text { mode }\end{array}$ & $\begin{array}{l}\text { Frequency/ } \\
\text { day }\end{array}$ & Frequency & $\begin{array}{l}\text { Duration } \\
\text { of Intake }\end{array}$ & $\begin{array}{l}\text { Observation } \\
\text { Time }\end{array}$ & $\begin{array}{l}\text { Assessed } \\
\text { Outcomes }\end{array}$ & $\begin{array}{l}\text { Primary } \\
\text { Outcome }\end{array}$ & $\begin{array}{l}\text { Outcomes } \\
\text { significantly } \\
\text { improved by } \\
\text { consumption } \\
\text { of probiotic }\end{array}$ \\
\hline $\begin{array}{l}\text { Invernici et al. } \\
\text { (64) }\end{array}$ & $\mathrm{RCT}$ & Periodontitis & 41 & 2 & Yes & Yes & $\begin{array}{l}\text { Bifidobacterium animalis. subsp. } \\
\text { lactis HNO19 }\end{array}$ & Lozenge & $1 \times 10^{9}$ & $2 \times$ daily & 30 days & 90 days & $\begin{array}{l}\text { CAL, PI, } \\
\text { BoP, PPD }\end{array}$ & CAL & CAL, PPD \\
\hline $\begin{array}{l}\text { Invernici et al. } \\
\text { (65) }\end{array}$ & $\mathrm{RCT}$ & Periodontitis & 30 & 2 & Yes & Yes & $\begin{array}{l}\text { Bifidobacterium animalis. subsp. } \\
\text { lactis HNO19 }\end{array}$ & Lozenge & $1 \times 10^{9}$ & $2 \times$ daily & 30 days & 90 days & $\begin{array}{l}\text { PI, GBI, } \\
\text { Adhesion }\end{array}$ & $\mathrm{PI}, \mathrm{GBI}$ & $\mathrm{Pl}, \mathrm{GBI}$ \\
\hline $\begin{array}{l}\text { Vivekananda } \\
\text { et al. (66) }\end{array}$ & $\mathrm{RCT}$ & Periodontitis & 30 & 2 & Yes & Yes & $\begin{array}{l}\text { Lactobacillus reuteri DSM } \\
\text { 17938/ATCC PTA } 5289\end{array}$ & Lozenge & $2 \times 10^{8}$ & $2 \times$ daily & 21 days & 42 days & $\begin{array}{l}\text { PI, Gl, GBI, } \\
\text { PPD, CAL }\end{array}$ & $\begin{array}{l}\text { not } \\
\text { specified }\end{array}$ & $\begin{array}{l}\text { PI, Gl, GBI, } \\
\text { CAL PPD }\end{array}$ \\
\hline $\begin{array}{l}\text { Teughels et al. } \\
\text { (67) }\end{array}$ & $\mathrm{RCT}$ & Periodontitis & 30 & 2 & Yes & Yes & $\begin{array}{l}\text { Lactobacillus reuteri DSM } \\
\text { 17938/ATCC PTA } 5289\end{array}$ & Lozenge & $2 \times 10^{8}$ & $2 \times$ daily & 84 days & 84 days & PPD, CAL & PPD & PPD, CAL \\
\hline Vicario et al. (68) & RCT & $\begin{array}{l}\text { Mild } \\
\text { periodontitis }\end{array}$ & 20 & 2 & No & No & $\begin{array}{l}\text { Lactobacillus reuteri DSM } \\
\text { 17938/ATCC PTA } 5289\end{array}$ & Lozenge & $2 \times 10^{8}$ & $1 \times$ daily & 30 days & 30 days & $\begin{array}{l}\text { PPD, PI, } \\
\text { BoP }\end{array}$ & $\begin{array}{l}\text { not } \\
\text { specified }\end{array}$ & PI, PPD, BoP \\
\hline Ince et al. (69) & $\mathrm{RCT}$ & Periodontitis & 30 & 2 & Yes & Yes & $\begin{array}{l}\text { Lactobacillus reuteri DSM } \\
\text { 17938/ATCC PTA } 5289\end{array}$ & Lozenge & $2 \times 10^{8}$ & $2 \times$ daily & 21 days & 360 days & $\begin{array}{l}\text { PPD, PI, Gl, } \\
\text { BOP }\end{array}$ & PPD & $\begin{array}{l}\text { PPD, Pl, Gl, } \\
\text { BOP }\end{array}$ \\
\hline Tekce et al. (70) & $\mathrm{RCT}$ & Periodontitis & 40 & 2 & Yes & Yes & $\begin{array}{l}\text { Lactobacillus reuteri DSM } \\
\text { 17938/ATCC PTA } 5289\end{array}$ & Lozenge & $2 \times 10^{8}$ & $2 \times$ daily & 21 days & 360 days & $\begin{array}{l}\text { PPD, PI, Gl, } \\
\text { BoP }\end{array}$ & , PPD & $\begin{array}{l}\text { PPD, Pl, Gl, } \\
\text { BoP }\end{array}$ \\
\hline $\begin{array}{l}\text { Theodoro et al. } \\
\text { (71) }\end{array}$ & $\mathrm{RCT}$ & $\begin{array}{l}\text { Periodontitis } \\
\text { smoking }\end{array}$ & 34 & 2 & Yes & Yes & $\begin{array}{l}\text { Lactobacillus reuteri DSM } \\
\text { 17938/ATCC PTA } 5289\end{array}$ & Lozenge & $2 \times 10^{8}$ & $2 \times$ daily & 21 days & 90 days & $\begin{array}{l}\text { PPD, BoP, } \\
\text { CAL }\end{array}$ & PPD & $\mathrm{BoP}$ \\
\hline Pelekos et al. (72) & 2) RCT & Periodontitis & 41 & 2 & Yes & Yes & $\begin{array}{l}\text { Lactobacillus reuteri DSM } \\
\text { 17938/ATCC PTA } 5289\end{array}$ & Lozenge & $2 \times 10^{8}$ & 2 × daily @ & 28 days & 180 days & $\begin{array}{l}\text { PPD, CAL, } \\
\text { BoP }\end{array}$ & PPD & None \\
\hline $\begin{array}{l}\text { Grusovin et al. } \\
\text { (73) }\end{array}$ & $\mathrm{RCT}$ & $\begin{array}{l}\text { Periodontitis } \\
\text { aftercare }\end{array}$ & 20 & 2 & Yes & Yes & $\begin{array}{l}\text { Lactobacillus reuteri DSM } \\
\text { 17938/ATCC PTA } 5289\end{array}$ & Lozenge & $2 \times 10^{8}$ & $2 \times$ daily & 84 days & 360 days & $\begin{array}{l}\text { PPD, CAL, } \\
\text { BoP }\end{array}$ & PPD & $\begin{array}{l}\text { PPD, CAL, } \\
\text { BoP }\end{array}$ \\
\hline Vohra et al. (74) & RCT & $\begin{array}{l}\text { Periodontitis } \\
\text { smokeless } \\
\text { tobacco }\end{array}$ & 127 & 4 & Yes & Yes & $\begin{array}{l}\text { Lactobacillus reuteri DSM } \\
\text { 17938/ATCC PTA } 5289\end{array}$ & Lozenge & $2 \times 10^{8}$ & $2 \times$ daily & 21 days & 180 days & $\begin{array}{l}\text { PPD, PI, } \\
\text { BoP, CAL }\end{array}$ & PPD & None \\
\hline $\begin{array}{l}\text { Laleman et al. } \\
\text { (75) }\end{array}$ & RCT & $\begin{array}{l}\text { Periodontitis } \\
\text { residual pockets }\end{array}$ & 39 & 2 & Yes & Yes & $\begin{array}{l}\text { Lactobacillus reuteri DSM } \\
\text { 17938/ATCC PTA } 5289\end{array}$ & Lozenge & $2 \times 10^{8}$ & $2 \times$ daily & 84 days & 168 days & $\begin{array}{l}\text { PPD, CAL, } \\
\text { PI, BoP }\end{array}$ & PPD & PPD \\
\hline Pelekos et al. (76) & ) $\mathrm{RCT}^{*}$ & Periodontitis & 40 & 2 & Yes & yes & $\begin{array}{l}\text { Lactobacillus reuteri DSM } \\
\text { 17938/ATCC PTA } 5289\end{array}$ & Lozenge & $2 \times 10^{8}$ & 2 × daily @ & 28 days & 180 days & PPD, CAL & PPD & $\begin{array}{l}\text { CAL in pockets } \\
\geq 5 \mathrm{~mm}\end{array}$ \\
\hline $\begin{array}{l}\text { Schlagenhauf } \\
\text { et al. (54) }\end{array}$ & $\mathrm{RCT} \#$ & $\begin{array}{l}\text { Periodontitis } \\
\text { smoking }\end{array}$ & 35 & 2 & No & No & $\begin{array}{l}\text { Lactobacillus reuteri DSM } \\
\text { 17938/ATCC PTA } 5289\end{array}$ & Lozenge & $2 \times 10^{8}$ & $2 \times$ daily & 42 days & 42 days & $\begin{array}{l}\text { BoP, CAL, } \\
\text { PPD }\end{array}$ & BoP & BoP, CAL, PPD \\
\hline Pudgar et al. (77) & RCT & Periodontitis & 40 & 2 & Yes & Yes & $\begin{array}{l}\text { Lactobacillus brevis CECT7489 } \\
\text { Lactobacillus plantarum } \\
\text { CECT7481 }\end{array}$ & Lozenge & $2 \times 10^{9}$ & $1 \times$ daily & 84 days & 84 days & $\begin{array}{l}\text { BoP, PPD, } \\
\text { PI, CAL, } \\
\text { GBI }\end{array}$ & $\begin{array}{l}\mathrm{PD}>4 \mathrm{~mm} \\
+\mathrm{BoP}\end{array}$ & $\mathrm{Bop} \S$ \\
\hline Iwasaki et al. (78) & RCT & $\begin{array}{l}\text { Periodontitis } \\
\text { aftercare }\end{array}$ & 39 & 2 & Yes & Yes & $\begin{array}{l}\text { Lactobacillus plantarum L-137 } \\
\text { (heat-killed) }\end{array}$ & Capsule & $10 \mathrm{mg}$ & $1 \times$ daily & 84 days & 84 days & $\begin{array}{l}\text { PPD, PI, Gl, } \\
\text { BoP }\end{array}$ & $\begin{array}{l}\text {, PPD > } \\
4 \mathrm{~mm}\end{array}$ & $\mathrm{PPD} \geq 4 \mathrm{~mm}$ \\
\hline Yuki et al. (79) & RCT & $\begin{array}{l}\text { Periodontitis } \\
\text { Intellect. } \\
\text { Disability }\end{array}$ & 23 & 2 & No & No & Lactobacillus rhamnosus L8020 & Milk yogurt & $1.5 \times 10^{9}$ & $1 \times$ daily & 90 days & 120 days & $\begin{array}{l}\text { PMA, Gl, } \\
\text { PPD }\end{array}$ & $\begin{array}{l}\text { not } \\
\text { specified }\end{array}$ & PMA \\
\hline Morales et al. (80) & ) RCT & Periodontitis & 28 & 2 & Yes & Yes & Lactobacillus rhamnosus SP-1 & Sachet & $2 \times 10^{7}$ & $1 \times$ daily & 84 days & 336 days & $\begin{array}{l}\text { CAL, PI, } \\
\text { BoP, PPD }\end{array}$ & CAL & None \\
\hline Morales et al. (81) & 1) RCT & Periodontitis & 47 & 3 & Yes & Yes & Lactobacillus rhamnosus SP-1 & Sachet & $2 \times 10^{7}$ & $1 \times$ daily & 84 days & 270 days & $\begin{array}{l}\text { CAL, PPD, } \\
\text { BoP }\end{array}$ & CAL & None \\
\hline $\begin{array}{l}\text { Laleman et al. } \\
\text { (82) }\end{array}$ & $\mathrm{RCT}$ & Periodontitis & 48 & 2 & Yes & Yes & $\begin{array}{l}\text { Streptococcus oralis KJ3 } \\
\text { Streptococcus uberis KJ2 } \\
\text { Streptococcus rattus JH145 }\end{array}$ & Lozenge & $3 \times 10^{8}$ & $2 \times$ daily & 84 days & 168 days & $\begin{array}{l}\text { PPD, CAL, } \\
\text { BoP, PI, Gl }\end{array}$ & PPD & None \\
\hline
\end{tabular}

BoP, Bleeding on Probing; CAL, Clinical Attachment Level; Gl, Gingival Index; GBI, Gingival Bleeding Index; PPD, Probing Pocket Depth; PMA, Papillary-Marginal-Attached-Index; PI, Plaque Index.

*Subanalysis of RCT published 2019 by the same authors. "Subanalysis of of 35 study participants out of a total of 72 displaying a PSR score $\geq 3$ at baseline. ${ }^{\circledR}$ Significantly negative impact on reduction of pockets $>4$ mm.

@ Study participants instructed to consume the lozenge prior to brushing their teeth. 


\section{Therapeutic Strategies}

The administration of the probiotic was either used as an adjunct to established measures of mechanical plaque control, i.e., oral hygiene training and/or professional mechanical plaque removal (PMPR) (47-50, 53, 55-57, 61-63), or was the only intervention performed $(51,52,54,58-60)$.

\section{Results}

Both therapeutic approaches led to diverging results. In some of the studies the administration of the probiotic as an adjunct to efficacious mechanical plaque control significantly enhanced the reduction of gingival inflammation $(48,60)$, while in others only minor or no significant benefits were observed $(47,49)$. In study cohorts with inadequate mechanical plaque control or in experimental gingivitis trials with cessation of oral hygiene measures, the results also were ambiguous. Kuru et al. (48) for example observed no difference in bacterial plaque mass or extent of gingival inflammation between study patients consuming a Bifidobacterium lactis subsp. animalis probiotic and the placeboconsuming controls during the first 4 weeks of the trial, while they were still maintaining an efficacious level of personal oral hygiene. After a 5-day cessation of personal hygiene efforts however, the resulting increase in plaque mass, gingival index and bleeding on probing scores observed in the probioticconsuming group was significantly lower than in the placeboconsuming controls. This was in contrast to a preceding study by Hallstrom et al. (50), who failed to detect any inhibitive impact of the consumption of a L.reuteri DSM 17938/ATCC PTA 5289 probiotic on the development of experimental gingivitis after the cessation of personal plaque control in healthy young volunteers with efficacious personal oral hygiene. However, in two other randomized clinical trials, evaluating the consumption of the identical L.reuteri probiotic by pregnant women (52) and navy sailors (54) with poor oral hygiene efficacy and evident clinical signs of established chronic gingivitis, its intake was accompanied by a significant and pronounced decrease of gingival inflammation when compared to the placeboconsuming controls, despite the lack of any concomitant attempt to improve oral hygiene efficacy.

\section{Probiotics in the Management of Periodontitis \\ Evaluated Study Cohorts}

The impact of probiotics administration in the management of periodontitis has also been evaluated in a wider spectrum of different patients, like systemically inconspicious non-smokers $(64,65,67,68,70)$, smokers $(54,71)$, smokeless tobacco users (74), periodontal recall patients $(73,78)$ or individuals with intellectual disabilities (79). They were also affected by diverging periodontal conditions like previously untreated periodontitis of varying severity, periodontitis aftercare $(73,78)$ or residual deep periodontal pockets (75).

\section{Evaluated Study Outcomes}

In 14 out of 20 RCTs assessed in this review the reduction of pocket depth was the primary study outcome $(66-78,82)$, followed by the gain of clinical attachment $(64,80,81)$ and the reduction of markers of inflammation, like gingival bleeding or bleeding on probing $(54,65,79)$.

\section{Therapeutic Strategies}

Predominantly the consumption of the probiotic was used as an adjunct to professional mechanical debridement and oral hygiene training. Only three trials evaluated the administration of the probiotic as the only therapeutic measure $(54,68,79)$.

\section{Results}

Similar to the situation in the gingivitis studies the results of the periodontitis trials do not display a uniform and consistent pattern. The majority of RCTs using the L. reuteri DSM 17938/ATCC PTA 5289 probiotic as an adjunct to mechanical biofilm and calculus removal reported a significant enhancement of pocket closure by the consumption of the probiotic and/or the reduction of periodontal inflammation compared to the intake of a placebo $(54,66-70,73,75,76)$. Nevertheless, there are some others studies, which either completely failed to detect any significant additional benefit of its use (72) or found only a very limited clinical usefulness in smokers and smokeless tobacco users $(71,74)$. In the case of the trial by Pelekos et al. (72) this might be possibly attributable to the reported administration scheme. The study participants were instructed to brush their teeth immediately after consuming the lozenge, which might have severely reduced dosage and contact time of the probiotic.

The adjunctive consumption of the other previously mentioned probiotics also led to diverging results. While studies evaluating the impact of $L$. rhamnosus SP-1 $(80,81)$ or a mixture of different probiotic strains of Streptococcus oralis, S. uberis and S. rattus (82) in untreated periodontitis patients failed to verify any additional clinical benefit of their regular consumption after scaling and root planing, the intake of Bifidobacterium lactis HN019-containing lozenges by contrast was accompanied by a significantly enhanced pocket closure and attachment gain as well as a reduction of periodontal inflammation when compared to the consumption of a placebo $(64,65)$. Furthermore, also the 12-week consumption of lozenges containing heat-killed Lactobacillus plantarum L-137 fragments, known to enhance Th1 helper cell-related immune functions in humans (83), resulted in a significant reduction of residual pocket depth in periodontal recall patients (78).

\section{Meta-Analyses and Systematic Reviews}

Confronted with this variety of partially contradictory results, conducting a meta-analysis or a systematic review of all previously described RCTs seems to be the most reasonable way to still be able to draw valid conclusions. A meta-analysis however is not a panacea for the analysis of any complex research question and may also only provide blurred answers when challenged with the task to compare study results being based on substantially diverging study protocols, patient cohorts and administered probiotics, as illustrated by the heterogeneity in the study protocols of the various trials listed in Tables 1, 2. Given the unique pattern of interferences with the host response and the competing microbiota associated with the use of each specific probiotic strain, it appears to be problematic to pool 
results obtained by the use of different probiotics in a metaanalysis. It is therefore not surprising, that the conclusions of currently available meta-analyses and systematic reviews on the use of probiotics in the control of gingivitis (84-86) or periodontitis (87-90) are not clear-cut, but vary considerably from "no additional benefit for pocket reduction" (87), to "limited, short term benefits in attachment gain or pocket closure of deeper pockets" (88-90) and "limited benefit for the reduction of gingival inflammation” (84-86).

\section{Controversies Over the Benefits of Probiotics-A Problem of Misunderstanding the Rationale for Their Use?}

An often-neglected aspect in the discussion about the benefits of probiotics in the management of gingivitis and periodontitis is the rationale for their use. As stated before, even the most recent guidelines for the therapy of gingivitis and periodontitis are still dominated by the principle of strict mechanical plaque control due to its proven efficacy in clinical practice. It is therefore no coincidence, that the authors of most probiotic trials used the probiotic only as an adjunct to those established mechanical plaque removal measures. Adding the consumption of a probiotic to properly performed mechanical plaque control in complying and systemically healthy patients however promises only little, if any additional short-term benefit to an already very efficacious therapeutic strategy. Efficacious however may not be confounded with cause-related, as the primary driving force for the development of inflammation-promoting oral bacterial dysbiosis is not insufficient mechanical plaque control (91). The actual and unique potential of probiotics for reducing inflammation on a systemic level becomes much more evident in gingivitis and periodontitis patients where the concept of strict mechanical plaque control fails or may not be applicable and in particular in those, who are concomitantly are also affected by altered systemic inflammatory responses for example heavy smokers, patients with systemic chronic-inflammatory diseases but also pregnant women (92). This is clearly reflected by the positive results of some aforementioned trials where the probiotic was given as the only therapeutic measure to individuals with established chronic gingivitis or periodontitis and habitual poor oral hygiene $(52,54,79)$. While the impact of mechanical plaque control on plaque-associated inflammation is always strictly local, many commonly used probiotics are known to be capable to downregulate inflammation also on a systemic level as described before (25). An elevated level of systemic inflammation, however, is suspected to be a major factor for

\section{REFERENCES}

1. Sender R, Fuchs S, Milo R. Revised estimates for the number of human and bacteria cells in the body. PLoS Biol. (2016) 14:e1002533. doi: 10.1371/journal.pbio.1002533

2. Tierney BT, Yang Z, Luber JM, Beaudin M, Wibowo MC, Baek C, et al. The landscape of genetic content in the gut and oral human microbiome. Cell Host Microbe. (2019) 26:283-95. doi: 10.1016/j.chom.2019.07.008 the initiation of periodontitis-promoting bacterial dysbiosis, as suggested by the significantly elevated incidence of periodontal disease in patients suffering from systemic chronic inflammatory conditions like diabetes type 2, rheumatoid arthritis or chronic kidney disease (39-41). As there is evidence from RCTs that the regular consumption of probiotics may also significantly improve the outcome of therapy in these chronic diseases (9395), their administration may have the potential to become an integral part of innovative, more cause-directed concepts for the management of gingivitis and periodontitis aiming at the primary prevention of bacterial dysbiosis by controlling inflammation not only locally, but also on a systemic level.

\section{Future Directions}

As a first future step however it seems to be mandatory, to rectify the evident current lack of commonly agreed study protocols and evaluation criteria for the assessment of the usefulness of probiotics administration in the control of gingivitis and periodontitis by a consensus conference with a broad participation of experts in the fields of periodontology, microbiology, immunology as well as gastroenterology and other subspecialties of internal medicine. This would provide the indispensable foundation for gaining sound and standardized study data from intervention trials, which would subsequently allow the performance of valid and meaningful comparisons and analyses.

\section{CONCLUSIONS}

At the present time the use of probiotics in the management of gingivitis and periodontitis is still a young and rapidly developing field with many unanswered questions including a lack of generally agreed recommendations regarding the selection of the appropriate probiotic, as well as the dosage and the duration of its use. Nevertheless, the use of probiotics with an efficacy record proven by randomized controlled clinical trials, may already represent a valuable addition to available therapy options, especially in clinical situations in which established concepts based on plaque control cannot be implemented with the required effectiveness.

\section{AUTHOR CONTRIBUTIONS}

US conceived and wrote the manuscript. YJ-S conducted the literature search and critically reviewed the final manuscript. All authors contributed to the article and approved the submitted version.

3. Salzberg SL. Open questions: How many genes do we have? BMC Biol. (2018) 16:94. doi: 10.1186/s12915-018-0564-x

4. Lloyd-Price J, Abu-Ali G, Huttenhower C. The healthy human microbiome. Genome Med. (2016) 8:51. doi: 10.1186/s13073-016-0307-y

5. West N, Chapple I, Claydon N, D'Aiuto F, Donos N, Ide M, et al. British society of $\mathrm{P}$, implant dentistry guideline group $\mathrm{P}$. BSP implementation of european s3 - level evidence-based treatment guidelines for stage i-iii periodontitis in uk clinical practice. J Dent. (2021) 106:103562. doi: 10.1016/j.jdent.2020.103562 
6. Sanz M, Herrera D, Kebschull M, Chapple I, Jepsen S, Beglundh T, et al. Treatment of stage I-III periodontitis-The EFP S3 level clinical practice guideline. J Clin Periodontol. (2020) 47:4-60. doi: 10.1111/jcpe.13290

7. Schwendicke F, Splieth CH, Bottenberg P, Breschi L, Campus G, Domejean S, et al. How to intervene in the caries process in adults: proximal and secondary caries? An EFCD-ORCA-DGZ expert Delphi consensus statement. Clin Oral Investig. (2020) 24:3315-21. doi: 10.1007/s00784-020-03431-0

8. Underhill DM, Gordon S, Imhof BA, Nunez G, Bousso P. Elie Metchnikoff (1845-1916): celebrating 100 years of cellular immunology and beyond. Nat Rev Immunol. (2016) 16:651-6. doi: 10.1038/nri.2016.89

9. Hill C, Guarner F, Reid G, Gibson GR, Merenstein DJ, Pot B, et al. Expert consensus document. The International Scientific Association for Probiotics and Prebiotics consensus statement on the scope and appropriate use of the term probiotic. Nat Rev Gastroenterol Hepatol. (2014) 11:506-14. doi: 10.1038/nrgastro.2014.66

10. Fijan S. Microorganisms with claimed probiotic properties: an overview of recent literature. Int J Environ Res Public Health. (2014) 11:4745-67. doi: 10.3390/ijerph110504745

11. Fukuda S, Toh H, Hase K, Oshima K, Nakanishi Y, Yoshimura K, et al. Bifidobacteria can protect from enteropathogenic infection through production of acetate. Nature. (2011) 469:543-7. doi: 10.1038/nature09646

12. Rosier BT, Moya-Gonzalvez EM, Corell-Escuin P, Mira A. Isolation and characterization of nitrate-reducing bacteria as potential probiotics for oral and systemic health. Front Microbiol. (2020) 11:555465. doi: 10.3389/fmicb.2020.555465

13. Dobson A, Cotter PD, Ross RP, Hill C. Bacteriocin production: a probiotic trait? Appl Environ Microbiol. (2012) 78:1-6. doi: 10.1128/AEM.05576-11

14. Geraldo BMC, Batalha MN, Milhan NVM, Rossoni RD, Scorzoni L, Anbinder AL. Heat-killed Lactobacillus reuteri and cell-free culture supernatant have similar effects to viable probiotics during interaction with Porphyromonas gingivalis. J Periodontal Res. (2020) 55:215-20. doi: 10.1111/jre.12704

15. Singh AK, Hertzberger RY, Knaus UG. Hydrogen peroxide production by lactobacilli promotes epithelial restitution during colitis. Redox Biol. (2018) 16:11-20. doi: 10.1016/j.redox.2018.02.003

16. Schwendicke F, Korte F, Dorfer CE, Kneist S, Fawzy El-Sayed K, Paris S. Inhibition of streptococcus mutans growth and biofilm formation by probiotics in vitro. Caries Res. (2017) 51:87-95. doi: 10.1159/000452960

17. Kim J, Kim J, Kim Y, Oh S, Song M, Choe JH, et al. Influences of quorumquenching probiotic bacteria on the gut microbial community and immune function in weaning pigs. Anim Sci J. (2018) 89:412-22. doi: 10.1111/asj.12954

18. Berstad A, Raa J, Midtvedt T, Valeur J. Probiotic lactic acid bacteria the fledgling cuckoos of the gut? Microb Ecol Health Dis. (2016) 27:31557. doi: 10.3402/mehd.v27.31557

19. Ohland CL, Macnaughton WK. Probiotic bacteria and intestinal epithelial barrier function. Am J Physiol Gastrointest Liver Physiol. (2010) 298:G807-19. doi: 10.1152/ajpgi.00243.2009

20. Paone P, Cani PD. Mucus barrier, mucins and gut microbiota: the expected slimy partners? Gut. (2020) 69:2232-43. doi: 10.1136/gutjnl-2020-322260

21. Poutahidis T, Kearney SM, Levkovich T, Qi P, Varian BJ, Lakritz JR, et al. Microbial symbionts accelerate wound healing via the neuropeptide hormone oxytocin. PLoS ONE. (2013) 8:e78898. doi: 10.1371/journal.pone.0078898

22. Varian BJ, Poutahidis T, DiBenedictis BT, Levkovich T, Ibrahim Y, Didyk E, et al. Microbial lysate upregulates host oxytocin. Brain Behav Immun. (2017) 61:36-49. doi: 10.1016/j.bbi.2016.11.002

23. Engevik MA, Ruan W, Esparza M, Fultz R, Shi Z, Engevik KA, et al. Immunomodulation of dendritic cells by Lactobacillus reuteri surface components and metabolites. Physiol Rep. (2021) 9:e14719. doi: 10.14814/phy2.14719

24. Liu Y, Fatheree NY, Dingle BM, Tran DQ, Rhoads JM. Lactobacillus reuteri DSM 17938 changes the frequency of Foxp3+ regulatory $\mathrm{T}$ cells in the intestine and mesenteric lymph node in experimental necrotizing enterocolitis. PLoS ONE. (2013) 8:e56547. doi: 10.1371/journal.pone.0056547

25. Yousefi B, Eslami M, Ghasemian A, Kokhaei P, Salek Farrokhi A, Darabi N. Probiotics importance and their immunomodulatory properties. J Cell Physiol. (2019) 234:8008-18. doi: 10.1002/jcp.27559

26. Han S, Lu Y, Xie J, Fei Y, Zheng G, Wang Z, et al. Probiotic gastrointestinal transit and colonization after oral administration: a long journey. Front Cell Infect Microbiol. (2021) 11:609722. doi: 10.3389/fcimb.2021.609722
27. Alforaidi S, Bresin A, Almosa N, Lehrkinder A, Lingstrom P. Oral colonisation after the administration of drops containing Lactobacillus reuteri. Oral Health Prev Dent. (2020) 18:1017-23. doi: 10.3290/j.ohpd.a45523

28. Kassim MA, Baijnath $H$, Odhav B. Effect of traditional leafy vegetables on the growth of lactobacilli and bifidobacteria. Int J Food Sci Nutr. (2014) 65:977-80. doi: 10.3109/09637486.2014.945155

29. Singh RK, Chang HW, Yan D, Lee KM, Ucmak D, Wong K, et al. Influence of diet on the gut microbiome and implications for human health. J Transl Med. (2017) 15:73. doi: 10.1186/s12967-017-1175-y

30. Suez J, Zmora N, Segal E, Elinav E. The pros, cons, and many unknowns of probiotics. Nat Med. (2019) 25:716-29. doi: 10.1038/s41591-019-0439-x

31. Borriello SP, Hammes WP, Holzapfel W, Marteau P, Schrezenmeir J, Vaara M, et al. Safety of probiotics that contain lactobacilli or bifidobacteria. Clin Infect Dis. (2003) 36:775-80. doi: 10.1086/368080

32. Boyle RJ, Robins-Browne RM, Tang ML. Probiotic use in clinical practice: what are the risks? Am J Clin Nutr. (2006) 83:1256-64. doi: 10.1093/ajcn/83.6.1256

33. Kosek MN, Penataro-Yori P, Paredes-Olortegui M, Lefante J, Ramal-Asayag C, Zamora-Babilonia M, et al. Safety of Lactobacillus reuteri DSM 17938 in healthy children 2-5 years of age. Pediatr Infect Dis J. (2019) 38:e178-e80. doi: 10.1097/INF.0000000000002267

34. Van den Nieuwboer M, Brummer RJ, Guarner F, Morelli L, Cabana M, Claasen E. The administration of probiotics and synbiotics in immune compromised adults: is it safe? Benef Microbes. (2015) 6:3-17. doi: 10.3920/BM2014.0079

35. van den Nieuwboer M, Brummer RJ, Guarner F, Morelli L, Cabana M, Claassen E. Safety of probiotics and synbiotics in children under 18 years of age. Benef Microbes. (2015) 6:615-30. doi: 10.3920/BM2014.0157

36. van den Nieuwboer $M$, Claassen E. Dealing with the remaining controversies of probiotic safety. Benef Microbes. (2019) 10:605-16. doi: 10.3920/BM2018.0159

37. van den Nieuwboer M, Claassen E, Morelli L, Guarner F, Brummer RJ. Probiotic and synbiotic safety in infants under two years of age. Benef Microbes. (2014) 5:45-60. doi: 10.3920/BM2013.0046

38. Haran JP, McCormick BA. Aging, frailty, and the microbiome-how dysbiosis influences human aging and disease. Gastroenterology. (2021) 160:507-23. doi: 10.1053/j.gastro.2020.09.060

39. Deschamps-Lenhardt S, Martin-Cabezas R, Hannedouche T, Huck O. Association between periodontitis and chronic kidney disease: Systematic review and meta-analysis. Oral Dis. (2019) 25:385-402. doi: 10.1111/odi.12834

40. Nascimento GG, Leite FRM, Vestergaard P, Scheutz F, Lopez R. Does diabetes increase the risk of periodontitis? A systematic review and meta-regression analysis of longitudinal prospective studies. Acta Diabetol. (2018) 55:653-67. doi: 10.1007/s00592-018-1120-4

41. Tang Q, Fu H, Qin B, Hu Z, Liu Y, Liang Y, et al. Possible link between rheumatoid arthritis and periodontitis: a systematic review and meta-analysis. Int J Periodontics Restorative Dent. (2017) 37:79-86. doi: 10.11607/prd.2656

42. Ebersole JL, Dawson DA. 3rd, Emecen Huja P, Pandruvada S, Basu A, Nguyen L, et al. Age and periodontal health - immunological view. Curr Oral Health Rep. (2018) 5:229-41. doi: 10.1007/s40496-018-0202-2

43. Rao SSC, Rehman A, Yu S, Andino NM. Brain fogginess, gas and bloating: a link between SIBO, probiotics and metabolic acidosis. Clin Transl Gastroenterol. (2018) 9:162. doi: 10.1038/s41424-018-0030-7

44. Quigley EMM, Pot B, Sanders ME. 'Brain Fogginess' and D-Lactic acidosis: probiotics are not the cause. Clin Transl Gastroenterol. (2018) 9:187. doi: 10.1038/s41424-018-0057-9

45. Papagaroufalis K, Fotiou A, Egli D, Tran LA, Steenhout P, A. Randomized double blind controlled safety trial evaluating d-Lactic acid production in healthy infants fed a Lactobacillus reuteri-containing formula. Nutr Metab Insights. (2014) 7:19-27. doi: 10.4137/NMI.S14113

46. McFarland LV, Evans CT, Goldstein EJC. Strain-specificity and diseasespecificity of probiotic efficacy: a systematic review and meta-analysis. Front Med (Lausanne). (2018) 5:124. doi: 10.3389/fmed.2018.00124

47. Alkaya B, Laleman I, Keceli S, Ozcelik O, Cenk Haytac M, Teughels W. Clinical effects of probiotics containing Bacillus species on gingivitis: a pilot randomized controlled trial. J Periodontal Res. (2017) 52:497-504. doi: $10.1111 /$ jre. 12415

48. Kuru BE, Laleman I, Yalnizoglu T, Kuru L, Teughels W. The influence of a bifidobacterium animalis probiotic on gingival health: A 
randomized controlled clinical trial. J Periodontol. (2017) 88:1115-23. doi: 10.1902/jop.2017.170213

49. Iniesta $M$, Herrera D, Montero E, Zurbriggen M, Matos AR, Marin MJ, et al. Probiotic effects of orally administered Lactobacillus reutericontaining tablets on the subgingival and salivary microbiota in patients with gingivitis. A randomized clinical trial. J Clin Periodontol. (2012) 39:736-44. doi: 10.1111/j.1600-051X.2012.01914.x

50. Hallstrom H, Lindgren S, Yucel-Lindberg T, Dahlen G, Renvert S, Twetman S. Effect of probiotic lozenges on inflammatory reactions and oral biofilm during experimental gingivitis. Acta Odontol Scand. (2013) 71:828-33. doi: 10.3109/00016357.2012.734406

51. Kraft-Bodi E, Jorgensen MR, Keller MK, Kragelund C, Twetman S. Effect of probiotic bacteria on oral candida in frail elderly. J Dent Res. (2015) 94:181S-6S. doi: 10.1177/0022034515595950

52. Schlagenhauf U, Jakob L, Eigenthaler M, Segerer S, Jockel-Schneider Y, Rehn M. Regular consumption of Lactobacillus reuteri-containing lozenges reduces pregnancy gingivitis: an RCT. J Clin Periodontol. (2016) 43:948-54. doi: $10.1111 /$ jcpe. 12606

53. Sabatini S, Lauritano D, Candotto V, Silvestre FJ, Nardi GM. Oral probiotics in the management of gingivitis in diabetic patients: a double blinded randomized controlled study. J Biol Regul Homeost Agents. (2017) 31:197-202.

54. Schlagenhauf U, Rehder J, Gelbrich G, Jockel-Schneider Y. Consumption of Lactobacillus reuteri-containing lozenges improves periodontal health in navy sailors at sea: A randomized controlled trial. J Periodontol. (2020) 91:1328-38. doi: 10.1002/JPER.19-0393

55. Ercan N, Olgun E, Kisa U, Yalim M. Effect of synbiotics in the treatment of smokers and non-smokers with gingivitis: randomized controlled trial. Aust Dent J. (2020) 65:210-9. doi: 10.1111/adj.12755

56. Lee JK, Kim SJ, Ko SH, Ouwehand AC, Ma DS. Modulation of the host response by probiotic Lactobacillus brevis CD2 in experimental gingivitis. Oral Dis. (2015) 21:705-12. doi: 10.1111/odi.12332

57. Montero E, Iniesta M, Rodrigo M, Marin MJ, Figuero E, Herrera D, et al. Clinical and microbiological effects of the adjunctive use of probiotics in the treatment of gingivitis: A randomized controlled clinical trial. J Clin Periodontol. (2017) 44:708-16. doi: 10.1111/jcpe.12752

58. Staab B, Eick S, Knofler G, Jentsch $\mathrm{H}$. The influence of a probiotic milk drink on the development of gingivitis: a pilot study. J Clin Periodontol. (2009) 36:850-6. doi: 10.1111/j.1600-051X.2009.01459.x

59. Slawik S, Staufenbiel I, Schilke R, Nicksch S, Weinspach K, Stiesch $M$, et al. Probiotics affect the clinical inflammatory parameters of experimental gingivitis in humans. Eur J Clin Nutr. (2011) 65:857-63. doi: $10.1038 /$ ejen.2011.45

60. Toiviainen A, Jalasvuori H, Lahti E, Gursoy U, Salminen S, Fontana M, et al. Impact of orally administered lozenges with Lactobacillus rhamnosus GG and Bifidobacterium animalis subsp. lactis BB-12 on the number of salivary mutans streptococci, amount of plaque, gingival inflammation and the oral microbiome in healthy adults. Clin Oral Investig. (2015) 19:77-83. doi: 10.1007/s00784-014-1221-6

61. Alanzi A, Honkala S, Honkala E, Varghese A, Tolvanen M, Soderling E. Effect of Lactobacillus rhamnosus and Bifidobacterium lactis on gingival health, dental plaque, and periodontopathogens in adolescents: a randomised placebo-controlled clinical trial. Benef Microbes. (2018) 9:593602. doi: 10.3920/BM2017.0139

62. Keller MK, Brandsborg E, Holmstrom K, Twetman S. Effect of tablets containing probiotic candidate strains on gingival inflammation and composition of the salivary microbiome: a randomised controlled trial. Benef Microbes. (2018) 9:487-94. doi: 10.3920/BM2017.0104

63. Kang MS, Lee DS, Lee SA, Kim MS, Nam SH. Effects of probiotic bacterium Weissella cibaria CMU on periodontal health and microbiota: a randomised, double-blind, placebo-controlled trial. BMC Oral Health. (2020) 20:243. doi: 10.1186/s12903-020-01231-2

64. Invernici MM, Salvador SL, Silva PHF, Soares MSM, Casarin R, Palioto DB, et al. Effects of Bifidobacterium probiotic on the treatment of chronic periodontitis: A randomized clinical trial. J Clin Periodontol. (2018) 45:1198210. doi: $10.1111 /$ jcpe. 12995

65. Invernici MM, Furlaneto FAC, Salvador SL, Ouwehand AC, Salminen S, Mantziari A, et al. Bifidobacterium animalis subsp lactis HN019 presents antimicrobial potential against periodontopathogens and modulates the immunological response of oral mucosa in periodontitis patients. PLOS ONE. (2020) 15:e0238425. doi: 10.1371/journal.pone.0238425

66. Vivekananda MR, Vandana KL, Bhat KG. Effect of the probiotic Lactobacilli reuteri (Prodentis) in the management of periodontal disease: a preliminary randomized clinical trial. J Oral Microbiol. (2010) 2:5344. doi: 10.3402 /jom.v2i 0.5344

67. Teughels W, Durukan A, Ozcelik O, Pauwels M, Quirynen M, Haytac MC. Clinical and microbiological effects of Lactobacillus reuteri probiotics in the treatment of chronic periodontitis: a randomized placebo-controlled study. $J$ Clin Periodontol. (2013) 40:1025-35. doi: 10.1111/jcpe.12155

68. Vicario M, Santos A, Violant D, Nart J, Giner L. Clinical changes in periodontal subjects with the probiotic Lactobacillus reuteri Prodentis: a preliminary randomized clinical trial. Acta Odontol Scand. (2013) 71:813-9. doi: $10.3109 / 00016357.2012 .734404$

69. Ince G, Gursoy H, Ipci SD, Cakar G, Emekli-Alturfan E, Yilmaz S. Clinical and biochemical evaluation of lozenges containing Lactobacillus reuteri as an adjunct to non-surgical periodontal therapy in chronic periodontitis. $J$ Periodontol. (2015) 86:746-54. doi: 10.1902/jop.2015.140612

70. Tekce M, Ince G, Gursoy H, Dirikan Ipci S, Cakar G, Kadir T, et al. Clinical and microbiological effects of probiotic lozenges in the treatment of chronic periodontitis: a 1-year follow-up study. J Clin Periodontol. (2015) 42:363-72. doi: $10.1111 /$ jcpe. 12387

71. Theodoro LH, Claudio MM, Nuernberg MAA, Miessi DMJ, Batista JA, Duque C, et al. Effects of Lactobacillus reuteri as an adjunct to the treatment of periodontitis in smokers: randomised clinical trial. Benef Microbes. (2019) 10:375-84. doi: 10.3920/BM2018.0150

72. Pelekos G, Ho SN, Acharya A, Leung WK, McGrath C, A. double-blind, paralleled-arm, placebo-controlled and randomized clinical trial of the effectiveness of probiotics as an adjunct in periodontal care. JClin Periodontol. (2019) 46:1217-27. doi: 10.1111/jcpe.13191

73. Grusovin MG, Bossini S, Calza S, Cappa V, Garzetti G, Scotti E, et al. Clinical efficacy of Lactobacillus reuteri-containing lozenges in the supportive therapy of generalized periodontitis stage III and IV, grade C: 1-year results of a double-blind randomized placebo-controlled pilot study. Clin Oral Investig. (2020) 24:2015-24. doi: 10.1007/s00784-019-03065-x

74. Vohra F, Bukhari IA, Sheikh SA, Albaijan R, Naseem M, Hussain M. Effectiveness of scaling and root planing with and without adjunct probiotic therapy in the treatment of chronic periodontitis among shamma users and non-users: A randomized controlled trial. J Periodontol. (2020) 91:1177-85. doi: 10.1002/JPER.19-0464

75. Laleman I, Pauwels M, Quirynen M, Teughels W. A dual-strain Lactobacilli reuteri probiotic improves the treatment of residual pockets: A randomized controlled clinical trial. J Clin Periodontol. (2020) 47:43-53. doi: 10.1111/jcpe.13198

76. Pelekos G, Acharya A, Eiji N, Hong G, Leung WK, McGrath C. Effects of adjunctive probiotic $L$. reuteri lozenges on S/RSD outcomes at molar sites with deep pockets. J Clin Periodontol. (2020) 47:1098-107. doi: 10.1111/jcpe.13329

77. Pudgar P, Povsic K, Cuk K, Seme K, Petelin M, Gaspersic R. Probiotic strains of Lactobacillus brevis and Lactobacillus plantarum as adjunct to non-surgical periodontal therapy: 3 -month results of a randomized controlled clinical trial. Clin Oral Investig. (2021) 25:1411-22. doi: 10.1007/s00784-020-03449-4

78. Iwasaki K, Maeda K, Hidaka K, Nemoto K, Hirose Y, Deguchi S. Daily intake of heat-killed Lactobacillus plantarum L-137 Decreases the probing depth in patients undergoing supportive periodontal therapy. Oral Health Prev Dent. (2016) 14:207-14. doi: 10.3290/j.ohpd.a36099

79. Yuki O, Furutani C, Mizota Y, Wakita A, Mimura S, Kihara T, et al. Effect of bovine milk fermented with Lactobacillus rhamnosus L8020 on periodontal disease in individuals with intellectual disability: a randomized clinical trial. $J$ Appl Oral Sci. (2019) 27:e20180564. doi: 10.1590/1678-7757-2018-0564

80. Morales A, Carvajal P, Silva N, Hernandez M, Godoy C, Rodriguez G, et al. Clinical Effects of Lactobacillus rhamnosus in Non-Surgical Treatment of Chronic Periodontitis: A Randomized Placebo-Controlled Trial With 1-Year Follow-Up. J Periodontol. (2016) 87:944-52. doi: 10.1902/jop.2016.150665

81. Morales A, Gandolfo A, Bravo J, Carvajal P, Silva N, Godoy C, et al. Microbiological and clinical effects of probiotics and antibiotics on nonsurgical treatment of chronic periodontitis: a randomized placebocontrolled trial with 9-month follow-up. J Appl Oral Sci. (2018) 26:e20170075. doi: 10.1590/1678-7757-2017-0075 
82. Laleman I, Yilmaz E, Ozcelik O, Haytac C, Pauwels M, Herrero ER, et al. The effect of a streptococci containing probiotic in periodontal therapy: a randomized controlled trial. J Clin Periodontol. (2015) 42:1032-41. doi: $10.1111 /$ jcpe. 12464

83. Hirose Y, Murosaki S, Yamamoto Y, Yoshikai Y, Tsuru T. Daily intake of heatkilled Lactobacillus plantarum L-137 augments acquired immunity in healthy adults. J Nutr. (2006) 136:3069-73. doi: 10.1093/jn/136.12.3069

84. Akram Z, Shafqat SS, Aati S, Kujan O, Fawzy A. Clinical efficacy of probiotics in the treatment of gingivitis: A systematic review and meta-analysis. Aust Dent J. (2020) 65:12-20. doi: 10.1111/adj.12733

85. Barboza EP, Arriaga PC, Luz DP, Montez C, Vianna KC. Systematic review of the effect of probiotics on experimental gingivitis in humans. Braz Oral Res. (2020) 34:e031. doi: 10.1590/1807-3107bor-2020.vol34.0031

86. Liu J, Liu Z, Huang J, Tao R. Effect of probiotics on gingival inflammation and oral microbiota: a meta-analysis. Oral Dis. (2021). doi: 10.1111/odi. 13861

87. Donos N, Calciolari E, Brusselaers N, Goldoni M, Bostanci N, Belibasakis GN. The adjunctive use of host modulators in non-surgical periodontal therapy. A systematic review of randomized, placebo-controlled clinical studies. J Clin Periodontol. (2020) 47:199-238. doi: 10.1111/jcpe. 13232

88. Ikram S, Hassan N, Raffat MA, Mirza S, Akram Z. Systematic review and meta-analysis of double-blind, placebo-controlled, randomized clinical trials using probiotics in chronic periodontitis. J Investig Clin Dent. (2018) 9:e12338. doi: $10.1111 /$ jicd. 12338

89. Martin-Cabezas R, Davideau JL, Tenenbaum H, Huck O. Clinical efficacy of probiotics as an adjunctive therapy to non-surgical periodontal treatment of chronic periodontitis: a systematic review and metaanalysis. J Clin Periodontol. (2016) 43:520-30. doi: 10.1111/jcpe. 12545

90. Vives-Soler A, Chimenos-Kustner E. Effect of probiotics as a complement to non-surgical periodontal therapy in chronic periodontitis: a systematic review. Med Oral Patol Oral Cir Bucal. (2020) 25:e161-7. doi: $10.4317 /$ medoral.23147

91. Hajishengallis G. Periodontitis: from microbial immune subversion to systemic inflammation. Nat Rev Immunol. (2015) 15:30-44. doi: $10.1038 /$ nri3785
92. Than NG, Hahn S, Rossi SW, Szekeres-Bartho J. Editorial: fetal-maternal immune interactions in pregnancy. Front Immunol. (2019) 10:2729. doi: 10.3389/fimmu.2019.02729

93. Bordalo Tonucci L, Dos Santos KM, De Luces Fortes Ferreira CL, Ribeiro SM, De Oliveira LL, Martino HS. Gut microbiota and probiotics: Focus on diabetes mellitus. Crit Rev Food Sci Nutr. (2017) 57:2296-309. doi: 10.1080/10408398.2014.934438

94. Eidi F, Poor-Reza Gholi F, Ostadrahimi A, Dalili N, Samadian F, Barzegari A. Effect of Lactobacillus Rhamnosus on serum uremic toxins (phenol and P-Cresol) in hemodialysis patients: A double blind randomized clinical trial. Clin Nutr ESPEN. (2018) 28:158-64. doi: 10.1016/j.clnesp.2018.08.010

95. Vaghef-Mehrabany E, Alipour B, Homayouni-Rad A, Sharif SK, AsghariJafarabadi M, Zavvari S. Probiotic supplementation improves inflammatory status in patients with rheumatoid arthritis. Nutrition. (2014) 30:430-5. doi: 10.1016/j.nut.2013.09.007

Conflict of Interest: US reports lecturing fees from BioGaia $\mathrm{AB}$ outside the submitted work.

The remaining author declares that the research was conducted in the absence of any commercial or financial relationships that could be construed as a potential conflict of interest.

Publisher's Note: All claims expressed in this article are solely those of the authors and do not necessarily represent those of their affiliated organizations, or those of the publisher, the editors and the reviewers. Any product that may be evaluated in this article, or claim that may be made by its manufacturer, is not guaranteed or endorsed by the publisher.

Copyright (c) 2021 Schlagenhauf and Jockel-Schneider. This is an open-access article distributed under the terms of the Creative Commons Attribution License (CC BY). The use, distribution or reproduction in other forums is permitted, provided the original author(s) and the copyright owner(s) are credited and that the original publication in this journal is cited, in accordance with accepted academic practice. No use, distribution or reproduction is permitted which does not comply with these terms. 